Check for updates

Cite this: J. Mater. Chem. B, 2019, 7, 3582

Received 28th November 2018, Accepted 30th April 2019

DOI: $10.1039 / c 8 t b 03135 \mathrm{e}$

rsc.li/materials-b

\section{Monitoring the death of single BaF3 cells under plasmonic photothermal heating induced by ultrasmall gold nanorods $\uparrow$}

\author{
Lisa Prisner $\ddagger$ Phillip Witthöft $\ddagger$ Lan Vi Ngoc Nguyen, Thomas Tsangas, \\ Tobias Gefken, Florentine Klaus, Christian Strelow, Tobias Kipp (DD * and Alf Mews (D)
}

\begin{abstract}
Gold nanorods are very suitable as energy converters for plasmonic photothermal therapy (PPTT) applications. These particles can be accumulated on cells by targeted or non-targeted delivery and subsequently internalized by the cells. Upon photoexcitation, the plasmonic particles create heat that can trigger cell-death mechanisms that are still not fully understood. Here, we used ultrasmall plasmonic gold nanorods, either non-targeted or targeted by functionalization with the aptamer AIR-3A. This aptamer specifically binds to the Interleukin-6 receptor expressed on the investigated BaF3 cell line. After photoexcitation of only individual cells, we monitored and investigated the evolution of cell death in real time on the single-cell level by tracking the temporal development of both the morphology and the absorbance of the cell, which changed due to the uptake of the dye trypan blue. We observed a swelling of the cell during the first few minutes, followed by a subsequent staining due to loss of membrane integrity, before cellular lysis took place. As the most notable difference between the use of targeted and non-targeted gold nanorods, the trypan blue staining period was considerably shorter when the targeted gold nanorods were used. Morphological changes of the cell after irradiation strongly suggest that necrosis is induced. The temporal delay between the start of the necrotic process and the beginning of cell staining indicates that the cell damage initially occurs in the inner cell compartments before the cell membrane is affected.
\end{abstract}

\section{Introduction}

One of the most promising emerging applications of nanostructures in cancer research is the use of plasmonic nanostructures like gold nanoparticles, -rods, or hollow spheres for plasmonic photothermal therapy (PPTT). Here, a temperature increase is induced via optical irradiation at the plasmonic absorption band of the nanostructures that are located on or in the tumor. This rise in tumor temperature can lead to cell death on its own, but also makes the tumor more susceptible to radiation or chemotherapy. ${ }^{1}$ Since non-malignant cells have unimpaired heat-shock protection mechanisms, cancerous cells are more susceptible to temperature rise. Therefore mild hyperthermia usually only induces very little damage in healthy cells. ${ }^{1}$ In an attempt to further reduce this damage to healthy tissue, temperature increase only in the cancerous tissue is desirable. For this goal, the use of targeted gold nanostructures, which can be specifically accumulated in the tumor by targeted delivery, shows great promise. The targeted delivery can be achieved

Institute of Physical Chemistry, University of Hamburg, Grindelallee 117, D-20146, Hamburg, Germany. E-mail: kipp@chemie.uni-hamburg.de; Tel: +49 40428388277 $\dagger$ Electronic supplementary information (ESI) available. See DOI: 10.1039/c8tb03135e \$ These authors contributed equally to this work. by decorating the gold nanostructures with biomolecules that specifically interact with surface receptors or molecules inherent to the desired cell type. ${ }^{2-11}$

In general, two types of cell death can be distinguished and it is of therapeutic significance which pathway is induced by the plasmonic photothermal effect. ${ }^{12}$ High temperature increase generally leads to cell death by necrosis, while temperature increase by only a few degrees Celsius induces apoptosis, the programmed cell death. ${ }^{1}$ While inducing apoptosis has the advantage that no inflammatory response is triggered as the apoptotic vesicles are phagocytosed, it has also been suggested that this pathway could lead to development of resistances. ${ }^{13}$ On the other hand necrosis is induced by inflicting substantial damage to key components of the cancerous cells such as the cell membrane or the mitochondria, ${ }^{6,13-15}$ which will inevitably lead to cell death. One of the downsides in the necrotic processes is that the cell is ultimately lysed completely and the cell fragments could lead to an inflammatory response in the organism. ${ }^{16}$ While both pathways may pose advantages and disadvantages depending on the application and the desired result, a clear understanding of the pathway induced in an experimental series is necessary. One simple way to distinguish these two pathways is to investigate the morphological changes in the cell. Necrotic cells initially swell due to the inability 
to regulate cell homeostasis after cell damage, which eventually leads to a loss of membrane integrity. ${ }^{12,13,17}$ Hence, the cell becomes leaky until it completely disintegrates and the cell components are released into the extracellular medium. Necrosis will lead to loss of membrane integrity early on in the process and can therefore easily be investigated by dye exclusion assays. ${ }^{18}$ In contrast, apoptotic cells shrink and the membrane forms blebs. The cell compartments are encapsulated in apoptotic vesicles, which are then phagocytosed.

In this study we investigated the cell death of individual cells induced by PPTT with microscopic techniques. Single cells, previously incubated with gold nanorods, were laser irradiated after which the transformation of the cell was monitored by optical microscopy. While the change in cell morphology was monitored directly, loss of membrane integrity was in parallel investigated by trypan-blue staining, as this dye cannot traverse the membrane of healthy cells.

In addition, we also explored the possibility of specific PPTT. Here we investigated the influence of targeted delivery to the Interleukin-6 (IL-6) receptor-carrying cells. For targeting the receptor, we chose the aptamer AIR-3A, which was specifically selected against the IL-6 receptor by Meyer et al. ${ }^{19}$ Upon binding to the receptor the aptamer can be taken up into the cell mediated by the receptor, ${ }^{19}$ carrying gold nanostructures or other cargo molecules. ${ }^{11,19,20}$

To transfer the optical excitation energy into local heat we chose elongated gold nanorods as they have fundamental advantages for photothermal therapy over spherical gold nanoparticles. Most notably, they exhibit an additional longitudinal plasmon resonance in the near-infrared (NIR) range of the spectrum, whereas spherical gold nanoparticles show a plasmon resonance in the visible region. ${ }^{21}$ As the depth of penetration of light into tissue is higher for light in the NIR region than in the visible region, the application of PPTT using elongated gold nanorods is more suitable. ${ }^{22,23}$ An additional factor in order to ascertain the suitability of gold nanostructures for PPTT is the absorption cross-section, which can be correlated to the size of the nanostructures. It has been shown that elongated gold nanorods with an effective radius (i.e. the radius of a sphere with a volume equaling that of the nanorod) larger than approximately $11 \mathrm{~nm}$ display high efficiency of scattering, thereby lowering the efficiency of absorption. ${ }^{23}$ As photothermal heating is induced by the excitation of the plasmon resonance, strong scattering is not desirable for this application. This is why we used ultrasmall nanorods exhibiting an effective radius of $5.6 \mathrm{~nm}$.

Many research groups have already demonstrated the advantageous plasmonic photothermal effect of gold nanorods for various applications. ${ }^{13,21,24-28}$ The microscopic study presented here allows to additionally investigate the mechanism of this effect and the subsequently induced cell death as well as the influence of the targeted delivery system mediated by the IL- 6 receptor. In our experiments, we find that for the targeted delivery system, the time until cell death during PPTT is significantly shorter than for non-targeted delivery. In addition, the morphological changes we observed during this process and the time-frame in which the cell membrane loses its integrity strongly suggest that in our case the plasmonic effect triggers cell death by necrosis as opposed to apoptosis. We observed a temporal delay until membrane integrity is lost after the necrotic process had already started. This indicates that initial damage to the cell is mainly due to the photothermal heating of intracellular compartments by internalized and membrane-bound gold nanorods. In our PPTT experiment, the cell membrane proves to be less affected than the inside of the cell.

\section{Experimental}

\subsection{Synthesis of ultrasmall gold nanorods}

All glassware was rinsed with aqua regia followed by deionized water before use. Ultrasmall gold nanorods were prepared using a tenfold scaled-up approach based on a route by Jia and coworkers. $^{29}$ The seed solution consists of chloroauric acid (0.75 mL, $0.01 \mathrm{M})$, cetrimonium bromide (CTAB/29.25 mL, $0.1 \mathrm{M})$, and freshly prepared and ice-cold sodium borohydride $(1.8 \mathrm{~mL}, 0.01 \mathrm{M})$. The mixture was vigorously stirred for two minutes and kept unstirred for aging (two hours) before use. To prepare the growth solution, a mixture of cetrimonium bromide (67.5 mL, 0.1 M), chloroauric acid (3.75 mL, 0.01 M), silver nitrate $(0.75 \mathrm{~mL}, 0.01 \mathrm{M})$, and hydrochloric acid $(1.5 \mathrm{~mL}$, $1 \mathrm{M}$ ) for adjusting the $\mathrm{pH}$ level was stirred at $30{ }^{\circ} \mathrm{C}$ for optimal stability conditions of the CTAB micelles. ${ }^{30}$ Very strong foaming should be avoided until this point. While increasing the level of stirring, ascorbic acid $(0.6 \mathrm{~mL}, 0.1 \mathrm{M})$ was injected, which results in disappearance of any color. Seed solution and growth solution were mixed in the ratio of $3: 7$. The solution was stirred quickly and subsequently kept unstirred overnight at $30{ }^{\circ} \mathrm{C}$.

For purification, the gold-nanorod solution was centrifuged twice. The first centrifugation step $\left(15000 \mathrm{rcf}, 30{ }^{\circ} \mathrm{C}, 60 \mathrm{~min}\right)$ leads to a reduction of the CTAB concentration. After resuspending the gold nanorods in water the second step (7000 rcf, $30{ }^{\circ} \mathrm{C}, 90 \mathrm{~min}$ ) leads to a particle size separation. ${ }^{31}$

\subsection{PEGylation of ultrasmall gold nanorods}

For experiments in the presence of living cells, CTAB ligands need to be removed from the gold nanorods, because of their cytotoxic character. ${ }^{32,33}$ For this purpose, adapting a method of Liu et al., ${ }^{34}$ CTAB was exchanged for a carboxyl- and thiol-terminated ethylene glycol (CT-PEG $\left.{ }_{12}\right)$ in the presence of bis( $p$-sulfonatophenyl)phenylphosphine (BSPP). In this functionalization process, $10 \mu \mathrm{L}$ of gold nanorod sample $(c=3.6 \mathrm{nM})$ were diluted in $140 \mu \mathrm{L}$ of Tris- $\mathrm{HCl}$ buffer with $0.05 \mathrm{wt} \%$ of polysorbate 20 (Tween 20). $\operatorname{BSPP}(23.20 \mu \mathrm{L}, 100 \mathrm{mM})$ and CT-PEG 12 (6.65 $\mu \mathrm{L}, 10 \mathrm{mM})$ were added and the solution was stirred vigorously. After $10 \mathrm{~min}$ of incubation, sodium chloride $(15 \mu \mathrm{L}, 5 \mathrm{M})$ was added and the stirring process was repeated. After $24 \mathrm{~h}$ at $30{ }^{\circ} \mathrm{C}$ the sample was then centrifuged (15000 rcf, RT, $30 \mathrm{~min}$ ). The precipitate was dissolved in $50 \mu \mathrm{L}$ of PBS-buffer solution.

\subsection{Characterization}

Size and optical properties of PEGylated ultrasmall gold nanorods were characterized using transmission electron microscopy (TEM) and UV-Vis absorption spectroscopy. The TEM measurements 
a

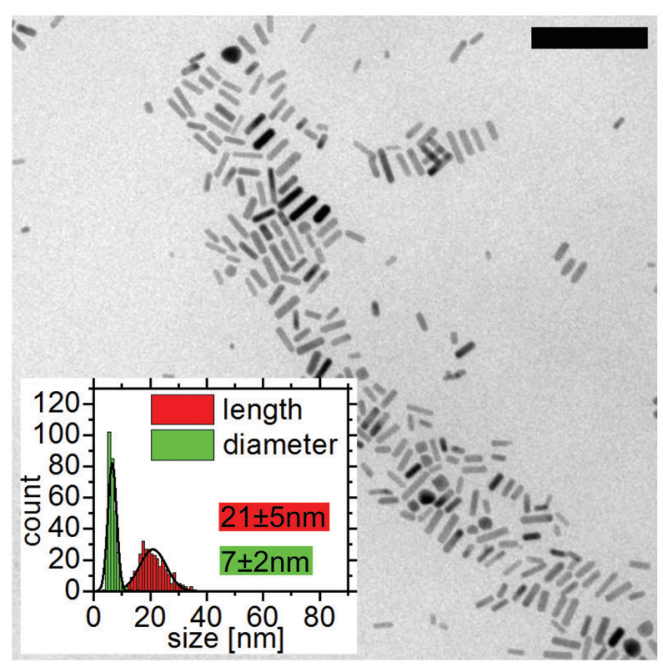

b

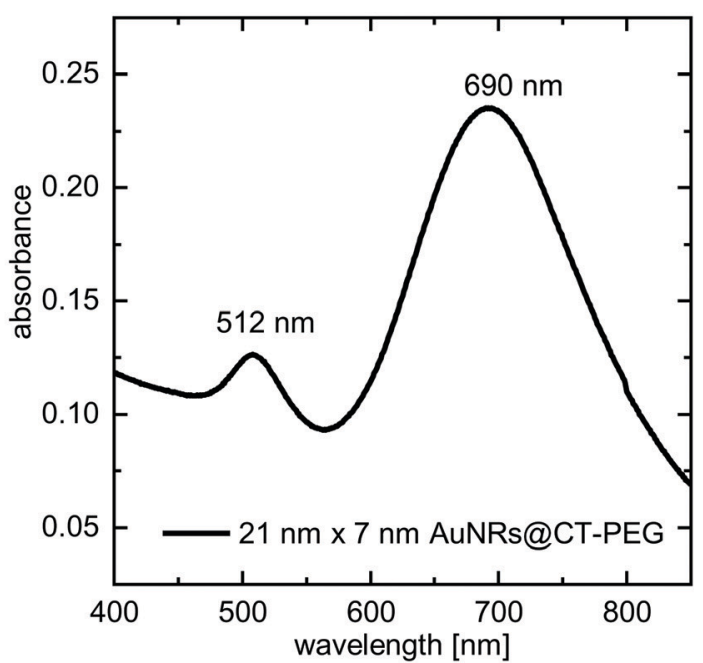

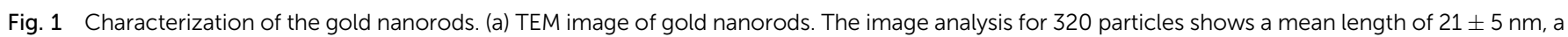

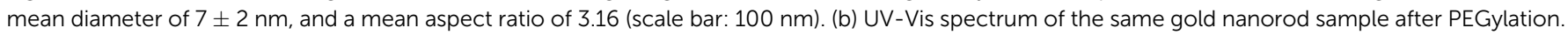
The longitudinal localized surface plasmon resonance (l-lspr) was measured to be $690 \mathrm{~nm}$.

were performed on a CM300 (Philips) with an acceleration voltage of $200 \mathrm{kV}$. A Cary 5000 spectrophotometer was used to perform the UV-Vis absorption spectroscopy. A TEM image, particle-size-distribution data, and an absorbance spectrum are given in Fig. 1.

\subsection{Introduction of an affinity molecule and formation of double-ligand layers on ultrasmall gold nanorods}

In order to introduce an affinity molecule specifically targeting the IL-6 receptor, a second ligand layer was attached to the carboxyl-terminated PEG layer, consisting of either pure amineand carboxyl-terminated $\mathrm{CA}-\mathrm{PEG}_{12}$ (carboxy-PEG $\mathrm{P}_{12}$-amine) or a mixed layer of $90 \% \mathrm{CA}^{-\mathrm{PEG}_{12}}$ and $10 \%$ of the IL-6 receptorspecific aptamer AIR-3A. The double-ligand-layer gold nanorods were synthesized via a classical EDC-NHS cross-coupling reaction. For that purpose, $30 \mu \mathrm{L}$ PEGylated gold nanorods $(c=8.4 \mathrm{nM})$ were diluted in $60 \mu \mathrm{L}$ MES buffer $(\mathrm{pH}=5.3)$. A $100 \times$ excess of EDC $(0.1 \mathrm{M})$ as well as a $200 \times$ excess of sulfo-NHS $(0.2 \mathrm{M})$ were added and incubated for 30 minutes at room temperature. The nanorods were centrifuged at $18000 \mathrm{rcf}$ for 30 minutes and the supernatant was discarded while the gold nanorods were resuspended in $50 \mu \mathrm{L}$ PBS buffer. A $12 \times$ excess of ligands relative to the hypothetical amount of binding sites on the nanorods (calculated from the nanoparticle concentration, surface area, and footprint of the ligands) was added of either only $\mathrm{CA}^{-\mathrm{PEG}_{12}}$ or the amine-terminated aptamer AIR-3A and CA-PEG 12 in a ratio of $1: 10$. The functionalized nanorods were purified by centrifugation at $15000 \mathrm{rcf}$ for 30 minutes at room temperature. The precipitate was dissolved in $50 \mu \mathrm{L}$ of PBS-buffer solution.

\subsection{Cell culture experiments}

IL-6 receptor carrying BaF3 cells were cultivated in DMEM (Dulbecco's modified Eagle's medium) completed with 10\% FCS (fetal calf serum) and 1\% $\mathrm{P} / \mathrm{S}$ (penicillin/streptomycin) at
$37{ }^{\circ} \mathrm{C}$ and $5 \% \mathrm{CO}_{2}$. For proliferation of the cells, the cytokine hIL-6 (human Interleukin-6) was added to the cell culture in a final concentration of $10 \mathrm{ng} \mathrm{mL}^{-1}$. Cells were passaged after reaching a cell density of 500000 cells per mL.

Cells were incubated with $1 \mathrm{nM}$ of ultrasmall gold nanorods for 30 minutes at $37{ }^{\circ} \mathrm{C}$ and $5 \% \mathrm{CO}_{2}$. After incubation, $0.4 \%$ trypan-blue solution was added to the cell suspension in a ratio of $1: 1$ before transfer into a Neubauer counting chamber. Single cells were irradiated with a laser and observed via optical microscopy every 10 seconds after the end of the irradiation.

For irradiation of the gold nanorods in the NIR region a supercontinuum white light laser source (SuperK Extreme, NKT Photonics) combined with a tunable single-line filter (SuperK Varia, NKT Photonics) operating at $690 \mathrm{~nm}$ with a bandwidth of $15 \mathrm{~nm}$ was used.

\subsection{Micrograph analysis for cell-death tracking in PPTT experiments}

For the evaluation of micrographs obtained with a light microscope in the PPTT experiments on BaF3 cells we tracked both, the visual change in morphology and also the contrast change because of interdiffusion of the trypan blue due to damage in the cell membrane.

Fig. 2 shows typical images of a single BaF3 cell incubated with gold nanorods and stained with trypan blue before and at certain times after laser irradiation. The images are based on the back-reflected red light; more precisely, they represent the red channel of the CCD camera. When a dye appears blue, it is absorbing mainly the red part of the white-light spectrum, thus the red channel of the CCD is linked to the absorption of light by trypan blue. Bright regions in the grey scale of the images corresponds to a small absorption and thus a large so-called red value (RV), as read out from the CCD. In reverse, dark pixels in the images correspond large absorption and a small RV. 
Before irradiation

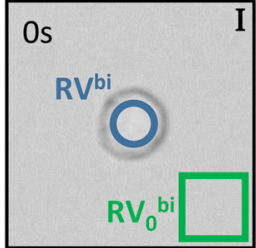

450 s
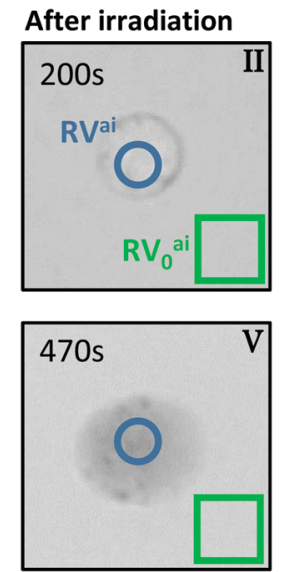

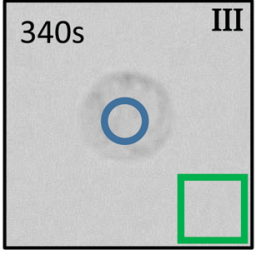

700 s 0
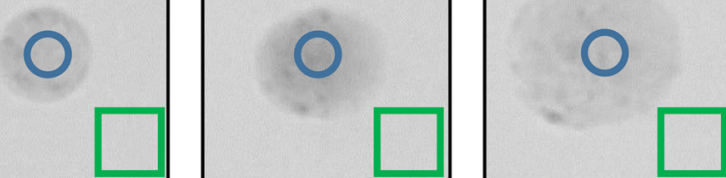

Fig. 2 Micrograph analysis for cell-death tracking. Typical micrographs of a single cell incubated with gold nanorods and stained with trypan blue before $(t=0 \mathrm{~s})$ and after certain times $(t=200 \mathrm{~s}, 340 \mathrm{~s}, 450 \mathrm{~s}, 470 \mathrm{~s}, 700 \mathrm{~s})$ after irradiation. Blue circles mark the regions in which the red values of the cell (RV) have been determined, green squares represent areas over which reference red values $\left(R V_{0}\right)$ were averaged.

In our micrograph analysis, the diameter of the cell is approximated by fitting a circle of variable diameter to the outer contrast line. The absorbance of the cell is calculated from:

$$
\text { Absorbance }=\log \left(\frac{R V_{0}^{\mathrm{ai}}}{R V^{\mathrm{ai}}}\right)-\log \left(\frac{R V_{0}^{\mathrm{bi}}}{R V^{\mathrm{bi}}}\right) .
$$

Here, $\mathrm{RV}^{\mathrm{bi}}$ and $\mathrm{RV}^{\mathrm{ai}}$ correspond to the red value before and at a certain time after irradiation, respectively. For each micrograph, these values have been averaged over a circular area in the cell (blue circles in Fig. 2). $\mathrm{RV}_{0}^{\mathrm{bi}}$ and $\mathrm{RV}_{0}^{\text {ai }}$ are reference red values for each $\mathrm{RV}^{\mathrm{bi}}$ and $\mathrm{RV}^{\mathrm{ai}}$, which are obtained for each micrograph by averaging over an area far away from the cell (green squares in Fig. 2). The introduction of these reference values into the calculation of the absorbance eliminates unwanted variations induced by fluctuations of the microscope illumination.

\section{Results \& discussion}

\subsection{Plasmonic photothermal effect of non-targeted ultrasmall gold nanorods on individual BaF3 cells}

In order to observe the plasmonic photothermal effect of gold nanorods, we synthesized nanorods with a length of $21 \mathrm{~nm}$ and a diameter of $7 \mathrm{~nm}$. Details on the synthesis, the characterization, and the PEGylation of the nanorods are given in the Methods section. Such ultrasmall gold nanorods show a plasmon resonance in the NIR region and are therefore potentially suitable for biological application in tissue. Their dimensions almost perfectly fit to the size that has previously been identified as being most effective for plasmonic photothermal therapy. ${ }^{35}$ This is because their small effective radius leads to a very high efficiency of absorption compared to their efficiency of scattering. ${ }^{23}$ Additionally, since the cellular uptake is also more effective ${ }^{36}$ and specific ${ }^{11}$ for smaller nanoparticles than for larger ones, our choice of ultrasmall gold nanorods should be ideally suited for photothermal applications. Immortalized murine BaF3 cells presenting the

IL-6 receptor on their surface were incubated with the PEGylated gold nanorods. These nanorods can only undergo non-specific interaction with the cells. After incubation and trypan-blue addition to the cell suspension, single cells were irradiated using laser light with a wavelength of $690 \mathrm{~nm}$. The size of the laser spot was determined to be $13.7 \mu \mathrm{m}$ and the power of the laser was set to $130 \mu \mathrm{W}$. This corresponds to a power density of $22 \mathrm{~W} \mathrm{~cm}^{-2}$, which is in a common range for PPTT. ${ }^{37-39}$ Details of the cell culture experiments including incubation, irradiation, and microscopy can be found in the Methods section.

Fig. 3(a) shows micrographs of an exemplary cell incubated with non-targeted PEG-coated gold nanorods captured before and every $10 \mathrm{~s}$ after the irradiation period of $60 \mathrm{~s}$. As it can be seen in the images, the cell shows morphological changes starting early after the irradiation. The size increases and after approximately $850 \mathrm{~s}$, the formerly sharp boundary between the cell and its surrounding begins to blur. The staining starts after approximately 5 minutes, indicating uptake of the dye as the membrane loses its integrity. The micrographs are further analyzed by determining in each image (i) a diameter of the cell and (ii) the degree of trypan-blue staining expressed by the absorbance of the cell. Details of the analysis are given in the Methods section.

Fig. 3(b) shows the development of both the cell size and the absorbance over time. It can be seen that the increase in the diameter starts immediately after the end of the irradiation and it quickly increases by almost $40 \%$ compared to its initial value until the diameter reaches a plateau at the time $t_{1} \approx 300 \mathrm{~s}$, at which the size stays almost constant for nearly $600 \mathrm{~s}$. Then, at $t_{2} \approx 850 \mathrm{~s}$, the diameter as determined from the micrographs drastically increases again. Regarding the absorbance, after the end of the irradiation, it increases only very slightly. After a delay time $t \approx 360 \mathrm{~s}>t_{1}$, the absorbance, which indicates the amount of trypan blue in the cell, strongly increases with a relatively constant slope $m$ until time $t_{2}$. After $t_{2}$, the absorbance sharply decreases again.

For comparison, Fig. 3(b) additionally shows the absorbance of a cell under the same experimental conditions but not incubated with gold nanorods. It can be seen that, in this case, the absorbance does not change significantly over time. The same is also true for the size of the cell (not shown here). This proves that in our experiments laser irradiation alone, in absence of plasmonic particles, does not lead to cell death. Furthermore, the toxicity of our ultrasmall gold nanorods alone without light irradiation was shown to be negligible (see ESI, $\dagger$ Fig. S1).

The results shown in Fig. 3(a) and (b) clearly indicate the cell death to occur by necrosis as opposed to apoptosis. First of all, the cell swelling that takes place until $t_{1}$ is typical for the necrotic process due to the cell's inability to uphold homeostasis. ${ }^{12,40}$ In the course of the necrotic process, eventually cellular lysis takes place, which we can identify to start at time $t_{2}$. In contrast, in an apoptotic process one would expect the cell first to shrink before the cell compartments are encapsulated in apoptotic vesicles. Also the staining of the cells by trypan blue in an early stage of the cell-death process is an indicator for a necrotic process. $^{12-14,17,41-43}$ 
a

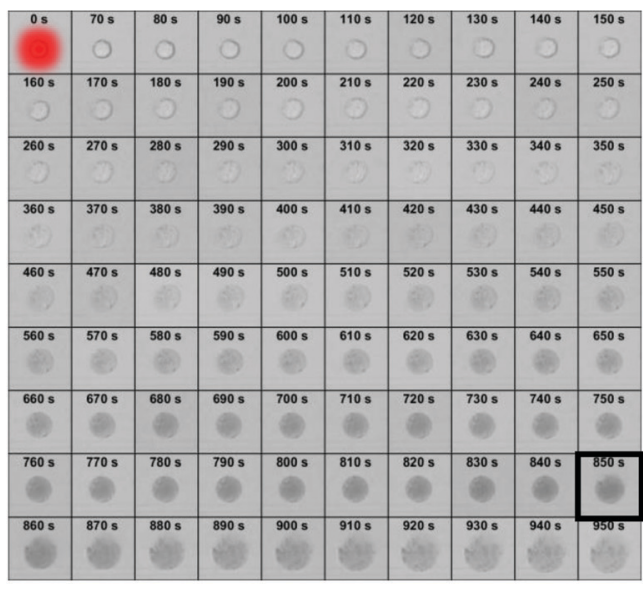

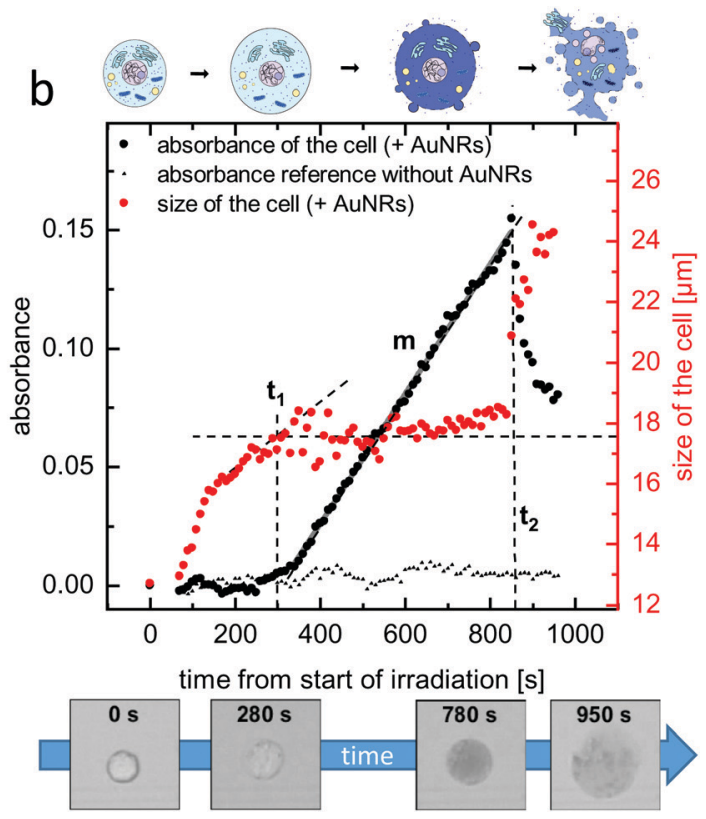

Fig. 3 Observation of the plasmonic photothermal effect of PEGylated ultrasmall gold nanorods on a single cell irradiated at $690 \mathrm{~nm}$ with $22 \mathrm{~W} \mathrm{~cm}^{-2}$ for

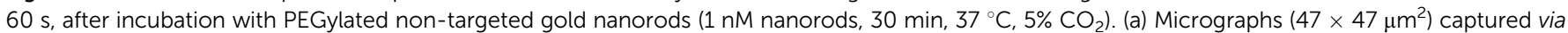
optical microscopy of the cell every $10 \mathrm{~s}$ starting after the end of the irradiation process. Laser-spot position is shown in red. Laser-spot dimensions not to scale. (b) Cell diameter and absorbance over time extracted from the micrographs in (a). Dashed lines are guides to the eye to determine times $t_{1}$ and $t_{2}$ (see text for more information). The top scheme depicts four key stages in the necrotic process corresponding to times $t=0, t<t_{1}, t_{1}<t<t_{2}$, and $t>t_{2}$, respectively.

Due to the morphological changes of the cell as well as the delay between cell swelling and cell staining, it seems likely that in our experiments the gold nanorods do not primarily damage the membrane directly. If this was the case, we would expect diffusion of the dye molecules into the damaged cell on a much faster timescale than the delay that can be seen in our experiments. Instead we propose that vital cell compartments are damaged by photothermal heating, eventually leading first to the initiation of the necrotic process followed by the loss of membrane integrity. It is well established in the literature that most nanoparticles, when endocytosed by a cell, eventually primarily accumulate in the lysosomes..$^{15,44-51}$ This is because the early endosomes, which contain the endocytosed material, are acidified and fuse with pre-lysosomal vesicles, before they turn into late endosomes and consequentially lysosomes. ${ }^{52} \mathrm{~A}$ break-out of the nanoparticles from the endosomes on this pathway is unlikely unless endosomal escape strategies are employed. Therefore, we hypothesize that the gold nanorods used in our experiments are located in the lysosomes after uptake. It is well known that the lysosomes are heavily involved in the necrotic cell-death mechanism when damage to the lysosomal membrane or lysosomal rupture occurs. Then a wide array of hydrolases is released into the cytosol that can degrade most cellular biomolecules and therefore initiate cell death. ${ }^{53}$ Consequently, we suppose that in our experiment the lysosomes are damaged photothermally via plasmons of the gold nanorods, which eventually initiates the necrotic cell death.

Summarizing this section, the sketch in the top of Fig. 3(b) depicts four key stages in the necrotic process each of which can be correlated to a micrograph in panel (a) as well as to values of the cell size and the cell absorbance at specific points in time. The first stage corresponds to the viable cell at $t=0 \mathrm{~s}$. The second stage represents cell swelling before strong staining at a time $t<t_{1}$. The third stage belongs to a stained cell with lost membrane integrity between $t_{1}$ and $t_{2}$. The forth stage is the cellular lysis after $t_{2}$.

\subsection{PPTT acceleration through higher delivery efficiency by the specific interaction between the ligand-coated ultrasmall gold nanorods and the cells}

The method outlined above was applied in order to compare the efficiency of PPTT using either non-targeted (PEGylated) or targeted (aptamer-functionalized) gold nanorods. Both ultrasmall gold nanorod variants feature a double PEG layer. The targeted nanorods have $10 \%$ of the outer PEG layer substituted by the IL- 6 receptor binding aptamer AIR-3A. IL- 6 receptorcarrying BaF3 cells were incubated with either non-targeted or targeted gold nanorods, trypan blue was added, and single cells were irradiated with laser light $(690 \mathrm{~nm}, 130 \mu \mathrm{W})$ for 60 seconds before micrographs were captured every $10 \mathrm{~s}$ (see ESI, $\dagger$ Fig. S2).

Fig. 4(a) shows the development of the cell size and absorbance over time for both the non-targeted PEGylated (top) and the targeted aptamer-functionalized (bottom) gold nanorods. Qualitatively, in both cases the curves resemble the ones shown in Fig. 3(b). Morphological changes start immediately after the end of the irradiation. The diameter increases due to cell swelling until it reaches a plateau at a time $t_{1}$. Here, $t_{1} \approx 200 \mathrm{~s}$ for both non-targeted and targeted gold nanorods. The diameter then 


\section{a}

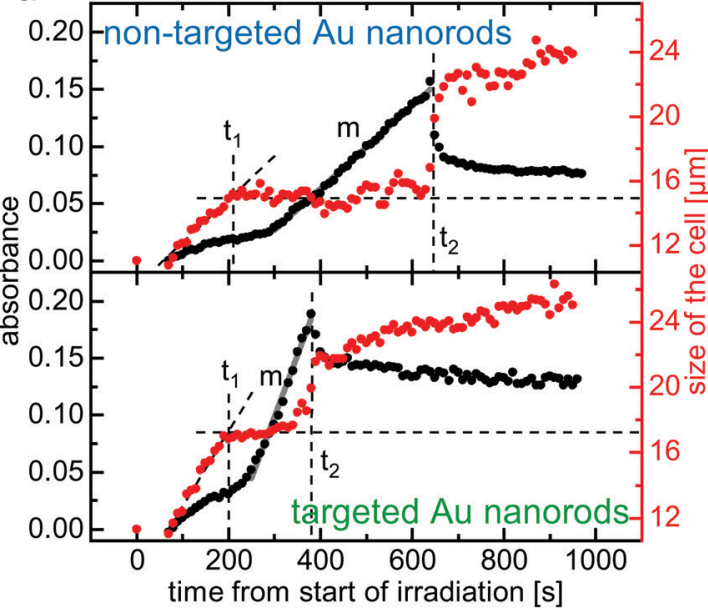

b

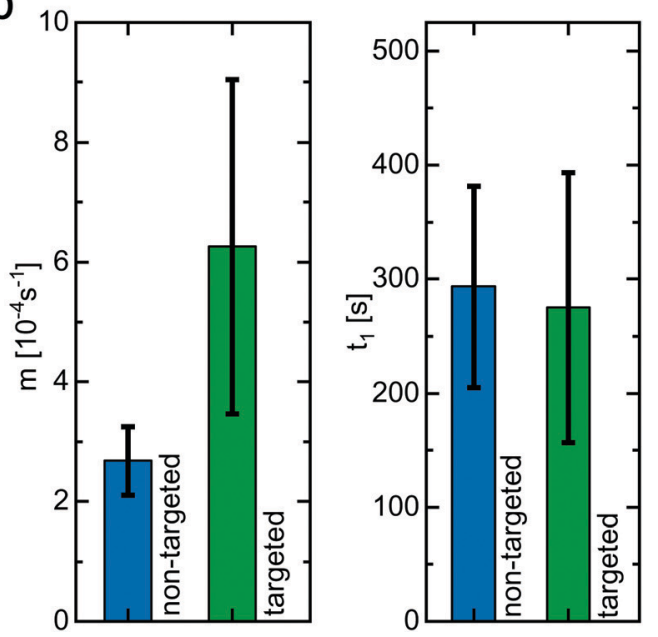

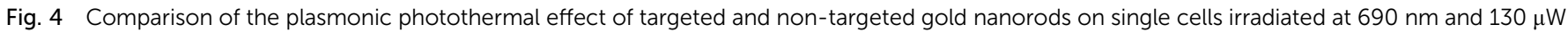

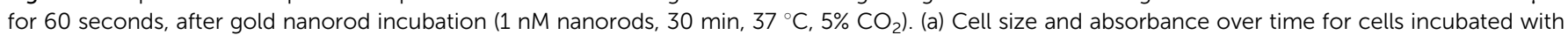

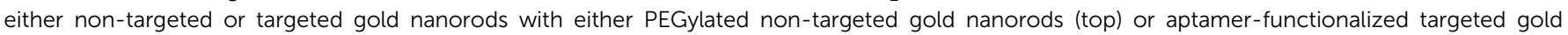

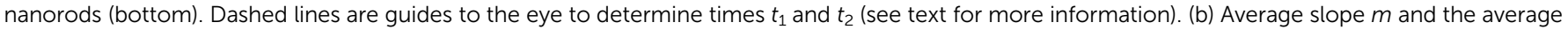
time $t_{1}$ for cells incubated with either targeted or non-targeted nanorods. The error bars represent standard deviations $(n=6)$.

remains constant until it drastically increases again at a time $t_{2}$, indicating cellular lysis. Here, $t_{2}$ is much smaller for the targeted gold nanorods ( $\approx 380 \mathrm{~s}$ ) than for the non-targeted ones ( $\approx 640 \mathrm{~s}$ ).

Regarding the absorbance, for both of these two cells a slight increase is observable even in the early stage of the process, for $t<t_{1}$. This increase, which did not occur for the cell analyzed in Fig. 3, could possibly be due to a small amount of trypan blue already entering the cells. However, taking into account the visual inspection of the actual micrographs from which the absorbance data was extracted (see ESI, $\dagger$ Fig. S2), it seems more likely that this increase stems from contrast changes due to the cell changing its morphology. This initial increase can be clearly differentiated from the rapid linear absorbance increase, which starts shortly after time $t_{1}$ and can clearly be attributed to the dye entering the cell, as we have described during the discussion of Fig. 3(b). The slope $m$ of this linear region, and therefore the rate at which the dye trypan blue enters the cell, is significantly different for the cells incubated with either nontargeted or targeted ultrasmall nanorods. The cell incubated with the targeted gold nanorods shows a much steeper incline of absorbance, indicating higher membrane permeability than for the non-targeted nanorods. We suggest that this is due to more extensive damage caused by photothermal heating as the targeted delivery allows accumulation of a higher concentration of gold nanorods in and on the target cell. The linear increase of the absorbance ends exactly at the time $t_{2}$, for which the size of the cell drastically jumps up. Here, lysis leads to a spread of the cellular contents, thereby decreasing the trypan blue concentration in the analyzed volume and hence leading to the measured absorbance drop after $t_{2}$.

The data presented in Fig. 3 and 4(a) was obtained from three different cells that belong to a series of in total twelve investigated cells, half of them incubated with non-targeted and half of them incubated with targeted gold nanorods.
Qualitatively, the data obtained from all cells is similar to the above discussed data. In particular, for each cell the parameters $t_{1}, t_{2}$, and $m$ could be clearly determined.

Fig. 4(b) shows the average slope $m$ and the average time $t_{1}$ for the cells incubated with either non-targeted or targeted nanorods. It can be seen that the average slope $m$ is indeed larger for the targeted gold nanorods. While the error bars are rather large due to variance from cell to cell in the same sample, the error bars do not overlap. This indicates that the difference between the two nanorod variants is of statistical significance and therefore strongly suggests a PPTT acceleration due to the targeted delivery system. As already mentioned, the larger slope $m$ for targeted nanorods indicate a higher membrane permeability, i.e., a higher degree of damage, caused by a higher concentration of gold nanorods in and on the cell. In contrast, the average time $t_{1}$, after which the cell swelling reaches a plateau, is similar for both non-targeted and targeted nanorods. The swelling process, which is due to loss of ion homeostasis, probably ends once the membrane starts to lose its integrity. At this time, the trypan blue dye molecules start to enter the cell and the staining begins. Our observations suggest that, at least for the different concentrations of gold nanorods as given by targeting and non-targeting in our experiment, the swelling time $t_{1}$ is determined by the time it takes for the cell on its necrotic pathway until its membrane starts to get harmed, which is in first approximation independent of the nanorod concentration.

Interestingly, regarding the error bars for the slope $m$ in Fig. 4(b), the variance for targeted nanorods is larger than in the non-targeted case. It can be speculated that this is due to additional variables that influence only the targeted delivery of the aptamer-functionalized gold nanorods and therefore the amount of gold nanorods accumulated in or on the cell during the incubation. While non-targeted gold nanorods can only interact with the cells via non-specific pathways, for the targeted 
gold nanorods non-specific as well as receptor-mediated, specific interaction is possible. The extent to which the non-specific interaction is supplemented by specific interaction for the targeted gold nanorods is dependent on additional parameters, such as level of receptor expression, that have no influence on the delivery of the non-targeted nanorod variant.

\section{Conclusion}

In this study, we investigated the plasmonic photothermal effect of elongated non-targeted and targeted gold nanorods in real time on single cells by monitoring morphological changes as well as the kinetics of trypan-blue staining (dye exclusion assay) of dying cells after laser irradiation. Analyzing the changes over time of morphology and of the absorbance leads to the following conclusions: in the case of our experiment, the photothermal heating caused by the irradiated gold nanorods leads to cell death by necrosis as opposed to apoptosis. The key stages in the necrotic process can be recognized in the captured micrographs and investigated in detail by analyzing the temporal changes of cell size and absorbance. A temporal delay between the onset of the necrotic process, evident by the morphological changes, and the subsequent loss of membrane integrity suggests that the initial damage to the cell occurs mainly inside the cell instead of on its membrane. Since endocytosed nanoparticles typically accumulate in the lysosomes, it can be assumed that photothermal heating in our experiment leads to damage to the lysosomal membrane and eventually to lysosomal rupture. This in turn can lead to the release of a wide array of hydrolases into the cytosol, which degrade a variety of essential biomolecules and therefore initiate cell death. We were able to show that cells incubated with targeted as opposed to non-targeted gold nanorods show a faster loss of membrane integrity due to more expansive damage to the cell via photothermal heating, since higher concentrations of gold nanorods can be accumulated in or on the cell when the targeted delivery is exploited.

\section{Conflicts of interest}

There are no conflicts to declare.

\section{Acknowledgements}

Financial support was received through the institutional strategy of the University of Hamburg. The work of Phillip Witthöft was funded by a scholarship from the PIER Helmholtz Graduate School. The authors thank Marina Mutas and Carola Schneider for helpful discussions.

\section{Notes and references}

1 D. K. Chatterjee, P. Diagaradjane and S. Krishnan, Ther. Delivery, 2011, 2, 1001-1014.

2 E. C. Dreaden, L. A. Austin, M. A. Mackey and M. A. El-Sayed, Ther. Delivery, 2012, 3, 457-478.
3 D. Bartczak, T. Sanchez-Elsner, F. Louafi, T. M. Millar and A. G. Kanaras, Small, 2011, 7, 388-394.

4 L. A. Dykman and N. G. Khlebtsov, Chem. Rev., 2014, 114, 1258-1288.

5 N. Li, T. Larson, H. H. Nguyen, K. V. Sokolov and A. D. Ellington, Chem. Commun., 2010, 46, 392-394.

6 A. M. Alkilany and C. J. Murphy, J. Nanopart. Res., 2010, 12, 2313-2333.

7 V. Peretz, M. Motiei, C. N. Sukenik and R. Popovtzer, J. At., Mol., Opt. Phys., 2012, 1-7.

8 B. B. Kasten, T. Liu, J. R. Nedrow-Byers, P. D. Benny and C. E. Berkman, Bioorg. Med. Chem. Lett., 2013, 23, 565-568.

9 H. Gao, Z. Yang, S. Zhang, S. Cao, S. Shen, Z. Pang and X. Jiang, Sci. Rep., 2013, 3, 2534.

10 R. Lévy, U. Shaheen, Y. Cesbron and V. Sée, Nano Rev., 2010, 1, 4889.

11 L. Prisner, N. Bohn, U. Hahn and A. Mews, Nanoscale, 2017, 9, 14486-14498.

12 N. Festjens, T. V. Berghe and P. Vandenabeele, Biochim. Biophys. Acta, Bioenerg., 2006, 1757, 1371-1387.

13 J. R. Melamed, R. S. Edelstein and E. S. Day, ACS Nano, 2015, 9, 6-11.

14 N. S. Abadeer and C. J. Murphy, J. Phys. Chem. C, 2016, 120, 4691-4716.

15 F. Zhang, D. Chen, Y. Wang, L. Zhang, W. Dong, J. Dai, C. Jin, X. Dong, Y. Sun, H. Zhao, K. Fan, H. Liu, B. Chen, H. Zou and W. Li, Nanomedicine, 2017, 12, 1575-1589.

16 S. L. Fink and B. T. Cookson, Infect. Immun., 2005, 73, 1907-1916.

17 Y. Pan, S. Neuss, A. Leifert, M. Fischler, F. Wen, U. Simon, G. Schmid, W. Brandau and W. Jahnen-Dechent, Small, 2007, 3, 1941-1949.

18 B. J. Marquis, S. A. Love, K. L. Braun and C. L. Haynes, Analyst, 2009, 134, 425.

19 C. Meyer, K. Eydeler, E. Magbanua, T. Zivkovic, N. Piganeau, I. Lorenzen, J. Grötzinger, G. Mayer, S. Rose-John and U. Hahn, RNA Biol., 2012, 9, 67-80.

20 S. Kruspe, C. Meyer and U. Hahn, Mol. Ther. - Nucleic Acids, 2014, 3, e143.

21 D. Bartczak, O. L. Muskens, S. Nitti, T. Sanchez-Elsner, T. M. Millar and A. G. Kanaras, Small, 2012, 8, 122-130.

22 J. Zhu, T. Gong, A. Kopwitthaya, R. Hu, W.-C. Law, I. Roy, H. Huang and K.-T. Yong, RSC Adv., 2013, 3, 12280.

23 P. K. Jain, K. S. Lee, I. H. El-Sayed and M. A. El-Sayed, J. Phys. Chem. B, 2006, 110, 7238-7248.

24 L. Tong, Y. Zhao, T. B. Huff, M. N. Hansen, A. Wei and J.-X. Cheng, Adv. Mater., 2007, 19, 3136-3141.

25 A. Bucharskaya, G. Maslyakova, G. Terentyuk, A. Yakunin, Y. Avetisyan, O. Bibikova, E. Tuchina, B. Khlebtsov, N. Khlebtsov and V. Tuchin, Int. J. Mol. Sci., 2016, 17, 1295.

26 Y. Ren, H. Qi, Q. Chen and L. Ruan, Int. J. Heat Mass Transfer, 2017, 106, 212-221.

27 S. Link and M. A. El-Sayed, Int. Rev. Phys. Chem., 2000, 19, 409-453.

28 T. B. Huff, L. Tong, Y. Zhao, M. N. Hansen, J.-X. Cheng and A. Wei, Nanomedicine, 2007, 2, 125-132. 
29 H. Jia, C. Fang, X.-M. Zhu, Q. Ruan, Y.-X. J. Wang and J. Wang, Langmuir, 2015, 31, 7418-7426.

30 N. C. Das, H. Cao, H. Kaiser, G. T. Warren, J. R. Gladden and P. E. Sokol, Langmuir, 2012, 28, 11962-11968.

31 F. Scaletti, C. S. Kim, L. Messori and V. M. Rotello, MethodsX, 2014, 1, e118-e123.

32 F. Schulz, W. Friedrich, K. Hoppe, T. Vossmeyer, H. Weller and H. Lange, Nanoscale, 2016, 8, 7296-7308.

33 C. J. Murphy, A. M. Gole, J. W. Stone, P. N. Sisco, A. M. Alkilany, E. C. Goldsmith and S. C. Baxter, Acc. Chem. Res., 2008, 41, 1721-1730.

34 K. Liu, Y. Zheng, X. Lu, T. Thai, N. A. Lee, U. Bach and J. J. Gooding, Langmuir, 2015, 31, 4973-4980.

35 M. A. Mackey, M. R. K. Ali, L. A. Austin, R. D. Near and M. A. El-Sayed, J. Phys. Chem. B, 2014, 118, 1319-1326.

36 N. Feliu, X. Sun, R. A. A. Puebla and W. J. Parak, Langmuir, 2017, 33, 6639-6646.

37 E. B. Dickerson, E. C. Dreaden, X. Huang, I. H. El-Sayed, H. Chu, S. Pushpanketh, J. F. McDonald and M. A. El-Sayed, Cancer Lett., 2008, 269, 57-66.

38 X. Huang, I. H. El-Sayed, W. Qian and M. A. El-Sayed, J. Am. Chem. Soc., 2006, 128, 2115-2120.

39 M. F. Tsai, S. H. G. Chang, F. Y. Cheng, V. Shanmugam, Y. S. Cheng, C. H. Su and C. S. Yeh, ACS Nano, 2013, 7, 5330-5342.

40 P. Syntichaki and N. Tavernarakis, EMBO Rep., 2002, 3, 604-609.
41 D. V. Krysko, T. V. Berghe, E. Parthoens, K. D'Herde and P. Vandenabeele, Methods Enzymol., 2008, 442, 307-341.

42 E. Brauchle, S. Thude, S. Y. Brucker and K. Schenke-Layland, Sci. Rep., 2014, 4, 1-9.

43 M. J. Akhtar, H. A. Alhadlaq, S. Kumar, S. A. Alrokayan and M. Ahamed, Arch. Toxicol., 2015, 89, 1895-1907.

44 L. Kou, J. Sun, Y. Zhai and Z. He, Asian J. Pharm. Sci., 2013, 8, 1-10. 45 A. Salvati, C. Åberg, T. dos Santos, J. Varela, P. Pinto, I. Lynch and K. A. Dawson, Nanomedicine, 2011, 7, 818-826.

46 J. A. Kim, C. Åberg, A. Salvati and K. A. Dawson, Nat. Nanotechnol., 2012, 7, 62-68.

47 G. C. Baltazar, S. Guha, W. Lu, J. Lim, K. Boesze-Battaglia, A. M. Laties, P. Tyagi, U. B. Kompella and C. H. Mitchell, PLoS One, 2012, 7, e49635.

48 M. Bourdenx, J. Daniel, E. Genin, F. N. Soria, M. Blancharddesce and E. Bezard, Autophagy, 2016, 12, 472-483.

49 X. Ma, Y. Wu, S. Jin, Y. Tian, X. Zhang, Y. Zhao, L. Yu and X.-J. Liang, ACS Nano, 2011, 5, 8629-8639.

50 N. Feliu, J. Hühn, M. V. Zyuzin, S. Ashraf, D. Valdeperez, A. Masood, A. H. Said, A. Escudero, B. Pelaz, E. Gonzalez, M. A. C. Duarte, S. Roy, I. Chakraborty, M. L. Lim, S. Sjöqvist, P. Jungebluth and W. J. Parak, Sci. Total Environ, 2016, 568, 819-828.

51 S. Guo and L. Huang, J. Nanomater., 2011, 1-12.

52 H. Hillaireau and P. Couvreur, Cell. Mol. Life Sci., 2009, 66, 2873-2896.

53 S. Aits and M. Jaattela, J. Cell Sci., 2013, 126, 1905-1912. 